\title{
1 Measuring G Protein Activation with Spectrally Resolved Fluorescence Fluctuation Spectroscopy
}

3 Authors: Daniel J. Foust ${ }^{1,2}, *$ David W. Piston ${ }^{1}$

4 1. Department of Cell Biology and Physiology, Washington University in St. Louis. St. Louis, MO, United

$5 \quad$ States.

6 2. Department of Biomedical Engineering, Washington University in St. Louis. St. Louis, MO, United

$7 \quad$ States.

$8 \quad *$ Corresponding Author: piston@wustl.edu 


\section{Abstract}

G protein-coupled receptor signaling has been posited to occur through either collision coupling or preassembled complexes with $\mathrm{G}$ protein transducers. To investigate the dynamics of $\mathrm{G}$ protein signaling, we introduce fluorescence covariance matrix analysis (FCMA), a novel implementation of fluorescence cumulant analysis applied to spectrally resolved fluorescence images. We labeled the GPCR, Ga, and $\mathrm{G} \beta \gamma$ units with distinct fluorescent protein labels and we applied FCMA to measure directly the complex formation during stimulation of dopamine and adrenergic receptors. To determine the prevalence of hetero-oligomers, we compared the GPCR data to those from control samples expressing three fluorescent protein labels with known stoichiometries. Interactions between G $\alpha$ and G $\beta \gamma$ subunits determined by FCMA were sensitive to stimulation with GPCR ligands. However, GPCR/G protein interactions were too weak to be distinguished from background. These findings support a collision coupling mechanism rather than pre-assembled complexes for the two GPCRs studied.

\section{Introduction}

Fluorescence fluctuation spectroscopy (FFS) is a set of statistical techniques used to extract physical parameters from fluorescence signals by using physical models of fluorescence detection (1). In biological applications, the most frequently measured parameters are diffusion coefficients, concentrations, and the molecular brightness of fluorescently labeled biomolecules (2-4). Imaging-FFS, by analyzing multidimensional fluorescence images, has become increasingly popular due to its potential to provide spatially resolved information (5-8). FFS is also used to analyze samples containing multiple chromophores and provide information about heteromeric molecular interactions $(9,10)$. Towards measuring an increasing number of molecular components, multicolor FFS has been expanded to utilize spectrally resolved detection. In these systems, a prism or diffraction grating is used to redirect photons 
onto an array of detectors so that the energy of incident photoelectrons is known more precisely than in

34

systems based on dichroic mirrors and multiple independent detectors (11-13). Recent developments have paired spectral detection with spectral unmixing techniques for better signal-to-noise ratios (SNRs) (14-16), and as many as four chromophores have been used simultaneously in live cell experiments (17). G protein coupled receptors (GPCRs) are physiologically vital cell surface receptors (18). Their downstream effects are predominantly mediated by $\mathrm{G}$ proteins (19-21). Trimeric $\mathrm{G}$ proteins are heterocomplexes composed of $\alpha, \beta$, and $\gamma$ subunits (22). Together, the $\beta$ and $\gamma$ subunits, form a single functional signaling unit and are not active when unpaired. Canonically, GPCRs catalyze the dissociation of $G \alpha \beta \gamma$ into active $G \alpha$ and $G \beta \gamma$ signaling units that can participate in downstream signaling events. The interactions between GPCRs and G proteins have been probed extensively using several fluorescencebased techniques including resonance energy transfer, bimolecular complementation, single molecule tracking, polarization, and FFS $(23,24)$.

An outstanding issue in GPCR signaling is the degree to which receptors and G proteins form stable complexes (25). Collision coupling models posit that GPCRs and G proteins move independently in the plasma membrane in the absence of stimulating ligands and are limited to transient interactions (26-28). Pre-assembly models assert that GPCRs and G proteins interact constitutively in stable complexes (29-31). Kinetic models have been developed to accommodate both signaling mechanisms (32), but experimental evidence to date has been contradictory regarding which mechanism is dominant (24). Multicolor FFS approaches are well-suited to address this problem because they enable us to observe the principal components of GPCR signal transduction simultaneously and in real time.

To investigate the mechanisms underlying GPCR activation, we introduce fluorescence covariance matrix analysis (FCMA), which is a new approach to analyzing spectrally resolved imaging data that provides detection and quantification of multi-chromic complexes. Additionally, FCMA 
provides a simple graphical interpretation of complex formation. FCMA is an extension of cumulant-

57 based approaches applied to spectrally resolved imaging data (8, 33-35). We applied FCMA to 23 protein monomers and dimers expressed on the plasma membrane. Using FCMA, we can detect and measure trimeric interactions without invoking higher order correlations (17). DRD2 and ADRA2A, whose exogenous ligands are dopamine and epinephrine, respectively. The Gai

63 subset of G proteins are involved in the inhibition of cAMP production through interactions with

64 adenylyl cyclase (36). In co-expression experiments with labeled Gai and G $\beta \gamma$ functional signaling units,

65 we monitored these three components during the signaling event. The $G \alpha / G \beta \gamma$ interactions were sensitive to stimulation with GPCR agonists. However, we did not observe ternary interactions between GPCRs, Gai, and G $\beta \gamma$ units, which is consistent with the absence of pre-assembled complexes.

\section{Results}

71 To calibrate FCMA as a tool for analyzing samples expressing multiple chromophores, we used the

72 monomeric plasma membrane protein CD86 as a scaffold for permutations of fluorescent heteromers.

73 Three fluorescent proteins were used, $\operatorname{mEGFP}(\mathrm{G}), \operatorname{mEYFP}(\mathrm{Y})$, and mCherry2 (R). Three fluorescent

74 monomers were analyzed, CD86-G, CD86-Y, and CD86-R, as well as five monomer/heteromer

75 combinations, CD86-G + CD86-Y + CD86-R (three monomers), R-CD86-Y-G (trimer), CD86-Y-G + CD86-R

76 (GY dimer with R monomer), CD86-G + R-CD86-Y (G monomer with YR dimer), and R-CD86-G + CD86-Y

77 (GR dimer with Y monomer) (Fig. 1). These combinations were expressed in HEK 293 cells and the

78 plasma membrane adjacent to the cover glass was imaged using confocal microscopy. 
Samples containing a single chromophore feature a single peak on the main diagonal of the

80

81

82

83

84 covariance matrix (Fig. $1 \mathrm{~A}-\mathrm{C}$ ). The location of this peak matches the position of the maximum signal in the detection spectrum (Fig. S1 C). When noninteracting species are present in a sample their contributions to the covariance matrix are additive (Fig. 1 D, F-H). When two chromophores interact and act as a single species this has a multiplicative effect on their contribution to the covariance matrix. Interacting chromophores with high spectral overlap produce a broadened peak, as in the case for $\mathrm{G}$ and $\mathrm{Y}$ (Fig. $1 \mathrm{E}-\mathrm{F}$ ). If interacting chromophores are well separated spectrally, their contribution to the covariance matrix produces lobes away from the main axis, as is the case for GR and YR interactions (Fig. $1 \mathrm{E}, \mathrm{G}-\mathrm{H})$. In the case of ternary interactions, there is both the more prominent, broad peak from the highly overlapping GY interactions and broader off axis lobes from concomitant GR and YR interactions

(Fig. $1 \mathrm{E})$.

\section{Distribution of Fluorescent Oligomer States by Fluorescence Covariance Matrix Analysis}

The covariance matrices and corresponding detection spectra from images of cells expressing three chromophores on CD86 were fit using a seven-component model accounting for the following species:
$\mathrm{G}, \mathrm{Y}, \mathrm{R}, \mathrm{GY}, \mathrm{GR}, \mathrm{YR}$, and GYR. For each component, an apparent number density is determined and the fractional distribution of each chromophore across different oligomer states is found (Fig. 2). The sum of fractional densities for each chromophore is unity. For the triple expression of monomeric proteins, CD86-G, CD86-Y, and CD86-R the largest fractions for each chromophore are observed in monomeric states (Fig. 2 A). We did observe some apparent GY and YR interactions which we take as the background levels for further experiments. GYR fractions were negligible.

In cells where the heterotrimer, R-CD86-Y-G was expressed, all three chromophores are found to be spread across several oligomer states (Fig. 2 B). This phenomenon can be understood by 
considering the existence of dark state fractions for each chromophore. A single protein will only contribute to the GYR (trimer) fraction if all of its constituent chromophores are correctly folded into the fluorescent state. Due to this effect, only small fractions ( 20-30\% per chromophore) appear in the trimeric state. Correspondingly, the dimeric states are populated by contributions from proteins with one chromophore in a dark state and the monomeric states are populated by contributions from proteins with two chromophores in dark states.

Data from cotransfections of dimer and monomer constructs produce similar results. For CD86-

112 fractions in $\mathrm{Y}$ and $\mathrm{R}$ monomeric states (Fig. $2 \mathrm{D}$ ). G is found almost exclusively in its monomeric state.

113 For R-CD86-G expressed with CD86-Y, G and R are split between their respective monomeric states and

114 GR interacting state, whereas $\mathrm{Y}$ almost exclusively found in its monomeric state (Fig. $2 \mathrm{E}$ ).

117 To compare with an established method, we processed the same datasets used for FCMA with spectrally 118 resolved raster image correlation spectroscopy (RICS). In contrast with FCMA, the 23 channel spectrally 119 resolved images must be unmixed prior to analysis into three single-color images, each corresponding to 120 a single chromophore (Fig. S1 B-D). Fitting model spatial auto- and cross-correlation functions allows for 121 the calculation of relative cross-correlation amplitudes, a readout of the interaction between pairs of

122 chromophores. Results from RICS analysis (Fig. 2 F) are in good agreement with those found with FCMA.

123 The data show very little interaction between chromophores in triple transfections of $G, Y$, and $R$

124 monomers. Images of the R-CD86-Y-G trimer exhibit strong, but not ideal, relative cross-correlations 
among the three possible pairings. For monomer/dimer coexpressions, CD86- $Y-G$ with CD86-R displays

127 displays strong GR interactions (Fig. 2 F). Conventional RICS analysis is limited to concomitant inferred. In contrast, ternary interactions can be quantified directly from covariance matrices (Fig. $1 \mathrm{E}$,

Fig. 2 B).

To express all three G proteins at physiologically appropriate and experimentally pragmatic levels, we modified a polycistronic construct introduced by Unen et al (37) to carry mCherry2 tags on GNB1 and GNG2 and an mEYFP(Q69K) tag on GNAI1 (Fig. S2). With this configuration, we labeled the two

136 functional $\mathrm{G}$ protein components with $\mathrm{Y}$ and $\mathrm{R}$. This construct was expressed in tandem with a $\mathrm{G}$ labeled 137 GPCR so that all three components of the GPCR signaling triplet could be monitored simultaneously.

138 The two Gai-coupled GPCRs studied here were DRD2 (dopamine) and ADRA2A (epinephrine).

141 interactions detected between GNAl1-Y and GPCRs G-DRD2 (Fig. 3 A-B) and G-ADRA2A (Fig. 3 C-D) are

142 comparable to background interaction levels found in control experiments featuring CD86 (Fig. 2 A).

143 There is significant interaction between GNAI1-Y and R-GNB1/R-GNG2 with $20 \%$ of $Y$ chromophores

144 being found in these interactions. The $Y$ and $Y R$ fractions are sensitive to GPCR stimulation by its native

145 ligand. When expressed with G-DRD2, the fraction of Y participating in YR interactions decreases from

$146 \sim 20 \%$ to $10 \%$ after stimulation with $100 \mu \mathrm{M}$ dopamine (Fig. 3 B). Similarly, when expressed with G- 
147 ADRA2A, the YR fraction decreases from 20\% to 10\% after stimulation with $30 \mu \mathrm{M}$ epinephrine (Fig. 3

148 D).

The distributions of G-DRD2 and G-ADRA2A are dominated by noninteracting fractions consisting of $\sim 90 \%$ of independent G chromophores (Fig. S3 A-B, Fig. S4 A-B). Apparent interactions with Y-GNAI1 and R-GNB1/R-GNG2 are comparable to background levels observed in control

152 experiments (Fig. 2 A) and trimer (GYR) fractions were negligible.

R-GNB1/R-GNG2 also appears almost entirely as noninteracting with the other chromophores

154 (>90\%) (Fig. S3 C-D, Fig. S4 C-D). The apparent asymmetry between the fraction of Y participating in YR 155 interactions and the fraction of R participating YR interactions comes from the relative expression levels 156 of these two chromophores. Because GNAI1-Y expression is dictated by an internal ribosome entry site 157 (Fig. S2), its expression is approximately three times lower than that of R-GNB1 and R-GNG2 (37).

We observe small relative changes in oligomer state distributions for G-GPCRs and R-GNB1/R-

159 GNG2 in response to GPCR stimulation. The GR fraction of R chromophores increases after dopamine 160 stimulation (Fig. S3 D), the GYR fraction of G chromophores decreases after epinephrine stimulation (Fig.

161 S4 B), and the GYR fraction of R chromophores also decreases after epinephrine stimulation (Fig. S4 D).

162 In each of these cases, the absolute oligomer fractions do not differ significantly from background levels

163 (Fig. 2) and are unlikely to represent biologically relevant findings. 
negligible relative cross-correlations for GY and GR pairings (Fig. 4). Like FCMA, we observe YR relative cross-correlations that are sensitive to GPCR stimulation (Fig. 4 B, D).

\section{Diffusion Coefficients by Spectrally Resolved RICS}

In contrast to FCMA, spectrally resolved RICS analysis also provides the apparent diffusion coefficient of each chromophore as an additional readout. While there are no statistically significant changes in the apparent diffusion coefficients for the GPCRs or G proteins in response to GPCR stimulation, there are distinct diffusivities among the components of the signaling cascade (Fig. 5). GPCRs, DRD2 and ADRA2A, are the least diffusive, averaging $~ 0.25 \mu \mathrm{m}^{2} / \mathrm{s}$. The R labelled GNB1/GNG2 component moves slightly faster than the receptors, averaging $\sim 0.5 \mu \mathrm{m}^{2} / \mathrm{s}$. The Y labelled GNAI1 is the most diffusive, $\sim 0.7 \mu \mathrm{m}^{2} / \mathrm{s}$, and shows a trend towards faster diffusion following stimulation of both GPCRs ( $p=0.071$ with G-DRD2 and $p=0.074$ for G-ADRA2A) (Fig. 5 B, D).

\section{Discussion}

We have demonstrated FCMA as a fluorescence fluctuation analysis tool suitable for multicolor imaging experiments in live cells. In this analysis, heteromeric combinations leave unique fingerprints on the covariance matrices calculated from spectrally resolved images (Fig. 1). The relative contributions of different oligomer states can be determined from fitting model functions and the resulting information tells us how chromophores are distributed across these states (Fig. 2).

FCMA is complementary to recent developments in spectrally resolved image correlation spectroscopy. FCMA and spectrally resolved RICS achieve many of the same goals. Both quantify the degree of interaction between two or more chromophores (Figs. 2-4). RICS, and other correlation 
function-based approaches, have the advantage of providing information about transport properties by outputting fitted diffusion coefficients (Fig. 5). However, as we show in this work, FCMA detects ternary interactions directly (Fig. 2 B), which offers a more robust and simpler computational procedure. higher order correlation functions which greatly increase the signal-to-noise requirements for the data and computational complexity $(17,38)$. Additionally, visual inspection of covariance matrices allows for the straightforward observation of complex formation that has an intuitive connection to the emission spectra (Fig. 1, Fig. S1 C). In practice, both analyses can be implemented in parallel with the same fluorescence imaging data.

The FMCA approach allows for simultaneous measurements of the three major components of the canonical GPCR/G-protein signaling pathway directly with fluorescence in live cells (Fig. 3, Fig. S3S4). These data are highly relevant to our mechanistic understanding of the signal propagation through GPCR/G protein pathways (23). The two predominant models of $\mathrm{G}$ protein activation are collisional coupling (26-28) and pre-assembled complexes (29-31). In collisional coupling models, GPCRs and G proteins have independent Brownian motions aside from their brief interactions when the GPCR is activated. Conversely, pre-assembly models posit that stable GPCR/G protein complexes are present with the components maintaining contact throughout the signaling processes. Biochemically, the 207 distinction between collisional coupling and pre-assembly models arises from the affinities of GPCR/G 
214 Although these data are consistent with a collisional coupling mechanism of GPCR signaling, we are

215 limited by the fidelity of the chromophores to radiative states (Fig. 2 B-E) (39). Recently, spectrally

216 resolved FFS for four chromophores has been demonstrated in live cell experiments, opening the door

217 for the expansion of the work shown here to include more components of the signaling cascade such as

218 downstream effectors (17).

\section{Methods}

\section{DNA Plasmids}

222 Subcloning to generate plasmids used in these studies was performed using standard molecular biology

223 techniques. Most experiments utilized In-Fusion cloning reagents from Takara Bio Inc or ig-Fusion

224 cloning reagents from Intact Genomics to facilitate subcloning unless noted otherwise. Reagents were

225 used following the manufacturers' protocols. Primers to generate linearized vectors and inserts are

226 listed in Table S1.

Monomeric and multimeric fluorescent controls were derived by modifying previously published plasmids designed to express CD86-EGFP (Addgene \# 133858) and mApple-CD86-EGFP (Addgene $\# 133860$ ) at the plasma membrane of mammalian cells (8). Parent plasmids were linearized using

230 inverse polymerase chain reactions to allow for insertions of fluorescent protein gene substitutes. Three

231 fluorescent proteins were used as labels in this work: mEGFP, mEYFP(Q69K), and mCherry2 (39-42),

232 abbreviated as G, $Y$, and $R$, respectively. Monomeric controls, CD86-G, CD86- $Y$, and CD86-R were

233 produced by making swaps against EGFP in CD86-EGFP. The dimeric controls R-CD86-G and R-CD86-Y

234 were produced by making dual swaps against mApple and EGFP in mApple-CD86-EGFP. To generate

235 CD86-Y-G and R-CD86-Y-G, an additional fluorescent protein site was introduced in the C-terminal linker

236 regions of CD86-G and R-CD86-G, respectively. mEGFP encoding inserts were amplified from G-DRD2 
237

described below. mEYFP(Q69K) was available from previous work in our lab (43). An mCherry2 donor plasmid (\#54563) was obtained from Addgene thanks to a generous donation by Michael Davidson.

A polycistronic construct to express $\mathrm{G}$ proteins GNAI1, GNB1, and GNG2 with the fluorescent protein labels used in control experiments was created by modifying a plasmid introduced by van Unen et al (37). The parent plasmid, GNB1-T2A-cpVenus-GNG2-IRES-GNAI1-mTurquoise2, was obtained from Addgene (\#69623). Both proteins of the G $\beta 1 \gamma 2$ functional unit were labeled with R. R-GNB1 was generated by swapping R against EGFP in EGFP-GNB1 described previously (Addgene \#133856) using restriction sites Agel and BsrGI (8). GNB1-T2A-cpVenus-GNG2-IRES-GNAI1-mTurquoise2 was linearized about GNB1 by digestion with Nhel and Sacl, and R-GNB1 was inserted. An N-terminal label of GNG2 was introduced by linearizing the parent plasmid about its cpVenus encoding region and inserting R. $Y$ was swapped against mTurquoise 2 in the parent plasmid in two steps. First $Y$ was swapped against mTurquoise2 in the monocistronic version of the plasmid, GNAl1-mTurqouise2 (Addgene \#69620), using restriction digestion at Agel sites to linearize the parent plasmid about mTurquoise2 and create GNAI1-Y by insertion of $\mathrm{Y}$ against mTurquoise2. BssHII and $\mathrm{Xmal}$ restriction sites were used to insert $\mathrm{Y}$ flanked by partial fragments of GNAl1 into the target vector using T4 ligation. A schematic of the final construct, RGNB1-T2A-R-GNG2-IRES-GNAI1-Y is shown in Figure S2.

G-DRD2 was created from EGFP-DRD2, from previous work $(8,44)$, by using site directed mutagenesis to introduce the A206K modification to EGFP (40). To create G-ADRA2A, G-DRD2 was linearized about its DRD2 encoding region. ADRA2A was amplified from ADRA2A-Tango, obtained from Addgene (\#66216) as a gift from Bryan Roth (45), and inserted against the position previously occupied by DRD2. All plasmids and their sequences will be made available via Addgene (https://www.addgene.org/Dave_Piston/). 
HEK293 cells were cultured in 1:1 Dulbecco's Modified Eagle's Medium/F-12 Ham with Glutamax+

supplemented with $10 \%$ fetal bovine serum albumin, penicillin, and streptomycin. Cells were incubated at $37 \mathrm{C}$ with $5 \% \mathrm{CO}_{2}$. Cells with passage number between 25 and 35 were used. For imaging, cells were transfected by electroporation. For a typical experiment, $1.5 \times 10^{6}$ cells were electroporated with 1-3 ug $30 \mathrm{~mm}$ diameters so that cells were at $50-75 \%$ confluency at the time of imaging. Electroporation was performed with eight $150 \mathrm{~V}$ pulses lasting $100 \mu$ s separated by 500 ms intervals. Cells were imaged $12-$ 36 hours after electroporation.

\section{Confocal microscopy}

271 All imaging experiments were conducting with a Zeiss LSM 880 confocal laser scanning microscope using

272 a 40x, 1.2 NA, C-Apochromat water immersion objective lens. $488 \mathrm{~nm}$ was used to excite $\mathrm{G}$ and $\mathrm{Y}$, and

$273561 \mathrm{~nm}$ was used to excite R. Typical laser intensities measured after the objective lens were $450 \mathrm{nW}$

274 for $488 \mathrm{~nm}$ and $1.3 \mu \mathrm{W}$ for $561 \mathrm{~nm}$. A 488/561 main beam splitter was used to separate excitation and

275 emission. For imaging of the basal plasma membrane for FCMA and spectrally resolved RICS analysis,

276 scanning was performed over $256 \times 256$ pixels spanning a $13.28 \times 13.28 \mu \mathrm{m}^{2}$ area, $50 \mathrm{~nm} / \mathrm{pixel}, 16.48$

277 us/pixel, and 45 frames per cell. The 32-bin Quasar spectral detector recorded photoelectron counts by

278 either photon counting or lambda mode in spectral bins of $8.9 \mathrm{~nm}$ spanning 490-695 $\mathrm{nm}$ (Figure S2).

Before imaging, cells were washed twice, and the growth buffer was replaced with Hanks'

280 Balanced Salt Solution (HBSS). During imaging cells were kept at $37 \mathrm{C}$ with $5 \% \mathrm{CO}_{2}$ using an incubated

281 stage. For GPCR stimulation experiments, the same cells were imaged before and after the addition of

282 the stimulus with approximately one minute of incubation between acquisitions. 
FCMA is an extension of multicolor fluorescence cumulant analysis applied to spectrally resolved cumulants, $\kappa_{[1,1]}(i, j)$, are equal to the covariance matrix for all pairs of channels, $(i, j)$.

$$
\begin{gathered}
\hat{\kappa}_{[1]}(i)=\left\langle F_{i}(x, y)\right\rangle_{(x, y) \in \mathrm{R}} \\
\hat{\kappa}_{[1,1]}(i, j)=\left\langle F_{i}(x, y) F_{j}(x+1, y)\right\rangle_{(x, y) \in \mathrm{R}}
\end{gathered}
$$

$F_{i}(x, y)$ denotes the fluorescence signal in photoelectrons at pixel $(x, y)$ in channel $i$. To select regions

292 of interest from images of basal plasma membranes we used a combination of polygonal selection and

293 intensity thresholds of blurred images described previously $(8,46)$. Region of interest selection was

294 performed on the spectrally unmixed images (Fig. S2 B-D) found for spectrally resolved RICS described

295 below. To avoid complications from crosstalk in spectral detection we used a single pixel offset in the 296 scanning axis (i.e. $(x+1))$ discussed in depth in previous work (8).

$$
\kappa_{[1,1]}(i, j)=\gamma_{2} \sum_{S}^{\text {species }} N_{S} \cdot \varepsilon_{S}{ }^{2} \cdot P_{S}{ }^{\{2\}}(i, j)
$$


$301 \gamma_{2}$ is a shape factor depending on the geometry of the detection volume. For this work we used $\gamma_{2}=$

3020.5 corresponding to a two-dimensional Gaussian detection volume (47). $P_{S}^{\{1\}}(i)$ for a species

303 consisting of a single chromophore is equal to that chromophore's detection spectrum normalized such

304 that its sum is unity. $P_{S}^{\{2\}}(i, j)$ is found from the outer product of $P_{S}^{\{1\}}(i)$ with itself, $P_{S}^{\{2\}}(i, j)=$

$305 P_{S}^{\{1\}}(i) \cdot P_{S}^{\{1\}}(j)$. When multiple chromophores are present in a species, $P_{S}{ }^{\{1\}}(i)$ is an average of its

306 component parts weighted by the brightnesses of its constitutive chromophores $(C)$ :

$$
P_{S}^{\{1\}}(i)=\frac{1}{\sum_{C} \varepsilon_{C}} \sum_{C} \varepsilon_{C} P_{C}^{\{1\}}(i)
$$

$P_{C}^{\{1\}}(i)$ are determined in control experiments where a single chromophore is expressed. A independent.

For the three chromophore experiments performed in this work, we fit a seven species model to the detection spectra and covariance matrices. The number density was allowed to vary for each species and the molecular brightness was linked across species so that 10 variables were determined

314 when fitting $\hat{\kappa}_{[1]}(i)$ and $\hat{\kappa}_{[1,1]}(i, j): N_{G}, N_{Y}, N_{R}, N_{G Y}, N_{G R}, N_{Y R}, N_{G Y R}, \varepsilon_{G}, \varepsilon_{Y}$, and $\varepsilon_{R}$. Fitting was

315 performed using Levenberg-Marquardt least squares minimization comparing experimentally determined $\hat{\kappa}_{[1]}$ and $\hat{\kappa}_{[1,1]}$ to theoretical values $\kappa_{[1]}$ and $\kappa_{[1,1]}(48,49)$.

$$
f_{x}=\frac{N_{x}}{N_{G}+N_{G Y}+N_{G R}+N_{G Y R}}
$$


Spectrally resolved RICS was implemented following the approach established by Schrimpf et al (16) was three frames.

332 from binary masks using the arbitrary region algorithm described by Hendrix et al (46). Correlation

333 functions were fit with a model function for two-dimensional diffusion:

$$
G(\xi, \psi)=G_{0}\left(1+\frac{4 D}{\omega_{0}^{2}}\left|\tau_{p x}\left(\xi-\xi_{0}\right)+\tau_{\ln } \psi\right|\right)^{-1} \exp \left(-\delta r^{2} \frac{\left(\left(\xi-\xi_{0}\right)^{2}+\psi^{2}\right)}{\omega_{0}^{2}+4 D\left|\tau_{p x}\left(\xi-\xi_{0}\right)+\tau_{l n} \psi\right|}\right)
$$
the pixel lag along the scanning axis that produces the greatest fitted amplitude, $G_{0}$.

341 fitted $\xi_{0}$ was greater than three this was treated as having a nonsignificant correlation amplitude so that $342 G_{0}=0$ for that experiment. This approach has the effect of ignoring spurious peaks at large pixel lags 
For the three chromophore experiments described in this work, spectrally resolved RICS

345 generates six correlation functions, three autocorrelation functions (GG, YY, RR) and three cross-

346 correlation functions (GY, GR, YR). Relative cross-correlations are calculated to quantify heteromeric

347 interactions:

$$
\text { Rel. } C C=\max \left\{\frac{G_{0}{ }^{i j}}{G_{0}{ }^{i i}}, \frac{G_{0}{ }^{i j}}{G_{0}{ }^{j j}}\right\}
$$

$349 \quad i$ and $j$ specify the chromophore/channel.

As quality control measures, only data with SNRs greater than three in all autocorrelation

351 functions and $G / Y$ mean fluorescence ratios bounded by $[1 / 10,10]$ were included. SNRs were calculated

352 using the method described by Schrimpf et al (16).

357 interquartile ranges. Unless indicated otherwise, statistical tests were two-sided paired t-tests

358 implemented with SciPy (50).

361 Analyses were performed using a series of IPython notebooks that are available at

362 https://github.com/d-foust/fcma. Source data are available upon request. 
The authors have no competing interests to declare.

366

367 Author contributions

368 DJF and DWP planned the research. DJF performed the experiments, completed the analysis, and wrote

369 the manuscript draft, which was edited by both authors.

371 Acknowledgements

372 DJF received support from National Institutes of Health training grants T32EB14855 and T32DK108742.

373 This research was supported by NIH grants R01DK123301 and R01DK115972 to DWP and by the

374 Washington University Center for Cellular Imaging supported in part by the Diabetes Research Center

375 (P30DK020579).

\section{References}

378

379

380
1. Elson, E.L. 2011. Fluorescence Correlation Spectroscopy: Past, Present, Future. Biophys. J. 101:28552870.

2. Qian, H., and E.L. Elson. 1990. Distribution of molecular aggregation by analysis of fluctuation moments. Proc. Natl. Acad. Sci. 87:5479-5483.

3. Chen, Y., J.D. Müller, P.T.C. So, and E. Gratton. 1999. The Photon Counting Histogram in Fluorescence Fluctuation Spectroscopy. Biophys. J. 77:553-567.

4. Magde, D., E. Elson, and W.W. Webb. 1972. Thermodynamic Fluctuations in a Reacting System--Measurement by Fluorescence Correlation Spectroscopy. Phys. Rev. Lett. 29:705-708.

5. Godin, A.G., S. Costantino, L.-E. Lorenzo, J.L. Swift, M. Sergeev, A. Ribeiro-da-Silva, Y.D. Koninck, and P.W. Wiseman. 2011. Revealing protein oligomerization and densities in situ using spatial intensity distribution analysis. Proc. Natl. Acad. Sci. 108:7010-7015. 
6. Sergeev, M., S. Costantino, and P.W. Wiseman. 2006. Measurement of Monomer-Oligomer Distributions via Fluorescence Moment Image Analysis. Biophys. J. 91:3884-3896.

7. Digman, M.A., R. Dalal, A.F. Horwitz, and E. Gratton. 2008. Mapping the Number of Molecules and Brightness in the Laser Scanning Microscope. Biophys. J. 94:2320-2332.

8. Foust, D.J., A.G. Godin, A. Ustione, P.W. Wiseman, and D.W. Piston. 2019. Two-Color Spatial Cumulant Analysis Detects Heteromeric Interactions between Membrane Proteins. Biophys. J. 117:1764-1777.

9. Chen, Y., M. Tekmen, L. Hillesheim, J. Skinner, B. Wu, and J.D. Müller. 2005. Dual-Color PhotonCounting Histogram. Biophys. J. 88:2177-2192.

10. Schwille, P., F.J. Meyer-Almes, and R. Rigler. 1997. Dual-color fluorescence cross-correlation spectroscopy for multicomponent diffusional analysis in solution. Biophys. J. 72:1878-1886.

11. Hwang, L.C., M. Leutenegger, M. Gösch, T. Lasser, P. Rigler, W. Meier, and T. Wohland. 2006. Prismbased multicolor fluorescence correlation spectrometer. Opt. Lett. 31:1310-1312.

12. Burkhardt, M., K.G. Heinze, and P. Schwille. 2005. Four-color fluorescence correlation spectroscopy realized in a grating-based detection platform. Opt. Lett. 30:2266-2268.

13. Previte, M.J.R., S. Pelet, K.H. Kim, C. Buehler, and P.T.C. So. 2008. Spectrally Resolved Fluorescence Correlation Spectroscopy Based on Global Analysis. Anal. Chem. 80:3277-3284.

14. Benda, A., P. Kapusta, M. Hof, and K. Gaus. 2014. Fluorescence spectral correlation spectroscopy (FSCS) for probes with highly overlapping emission spectra. Opt. Express. 22:2973-2988.

15. Felekyan, S., S. Kalinin, H. Sanabria, A. Valeri, and C.A.M. Seidel. 2012. Filtered FCS: Species Autoand Cross-Correlation Functions Highlight Binding and Dynamics in Biomolecules. ChemPhysChem. 13:1036-1053.

16. Schrimpf, W., V. Lemmens, N. Smisdom, M. Ameloot, D.C. Lamb, and J. Hendrix. 2018. Crosstalk-free multicolor RICS using spectral weighting. Methods. 140-141:97-111.

17. Dunsing, V., A. Petrich, and S. Chiantia. 2021. Multi-color fluorescence fluctuation spectroscopy in living cells via spectral detection. eLife. 10:e69687.

18. Lefkowitz, R.J. 2004. Historical review: a brief history and personal retrospective of seventransmembrane receptors. Trends Pharmacol. Sci. 25:413-422.

19. Sutherland, E.W., and T.W. Rall. 1958. Fractionation and characterization of a cyclic adenine ribonucleotide formed by tissue particles. J. Biol. Chem. 232:1077-1091.

20. Gilman, A.G. 1987. G proteins: transducers of receptor-generated signals. Annu. Rev. Biochem. 56:615-649.

21. Rodbell, M. 1980. The role of hormone receptors and GTP-regulatory proteins in membrane transduction. Nature. 284:17-22. 
22. Bokoch, G.M., T. Katada, J.K. Northup, M. Ui, and A.G. Gilman. 1984. Purification and properties of the inhibitory guanine nucleotide-binding regulatory component of adenylate cyclase. J. Biol. Chem. 259:3560-3567.

23. Calebiro, D., Z. Koszegi, Y. Lanoiselée, T. Miljus, and S. O’Brien. 2021. G protein-coupled receptor-G protein interactions: a single-molecule perspective. Physiol. Rev. 101:857-906.

24. Bondar, A., and J. Lazar. 2017. The G protein Gi1 exhibits basal coupling but not preassembly with $\mathrm{G}$ protein-coupled receptors. J. Biol. Chem. 292:9690-9698.

25. Oldham, W.M., and H.E. Hamm. 2008. Heterotrimeric G protein activation by G-protein-coupled receptors. Nat. Rev. Mol. Cell Biol. 9:60-71.

26. 2005. Dynamics of receptor/G protein coupling in living cells. EMBO J. 24:4106-4114.

27. Orly, J., and M. Schramm. 1976. Coupling of catecholamine receptor from one cell with adenylate cyclase from another cell by cell fusion. Proc. Natl. Acad. Sci. 73:4410-4414.

28. Tolkovsky, A.M., and A. Levitzki. 1978. Mode of coupling between the $\beta$-adrenergic receptor and adenylate cyclase in turkey erythrocytes. Biochemistry. 17:3795-3810.

29. De Lean, A., J.M. Stadel, and R.J. Lefkowitz. 1980. A ternary complex model explains the agonistspecific binding properties of the adenylate cyclase-coupled beta-adrenergic receptor. J. Biol. Chem. 255:7108-7117.

30. Tolkovsky, A.M., and A. Levitzki. 1978. Coupling of a single adenylate cyclase of two receptors: adenosine and catecholamine. Biochemistry. 17:3811-3817.

31. Lohse, M.J., K.N. Klotz, and U. Schwabe. 1991. Mechanism of A2 adenosine receptor activation. I. Blockade of A2 adenosine receptors by photoaffinity labeling. Mol. Pharmacol. 39:517-523.

32. Weiss, J.M., P.H. Morgan, M.W. Lutz, and T.P. Kenakin. 1996. The Cubic Ternary Complex ReceptorOccupancy Model I. Model Description. J. Theor. Biol. 178:151-167.

33. Müller, J.D. 2004. Cumulant Analysis in Fluorescence Fluctuation Spectroscopy. Biophys. J. 86:39813992.

34. Wu, B., Y. Chen, and J.D. Müller. 2006. Dual-Color Time-Integrated Fluorescence Cumulant Analysis. Biophys. J. 91:2687-2698.

35. Hur, K.-H., Y. Chen, and J.D. Mueller. 2016. Characterization of Ternary Protein Systems In Vivo with Tricolor Heterospecies Partition Analysis. Biophys. J. 110:1158-1167.

36. Wettschureck, N., and S. Offermanns. 2005. Mammalian G proteins and their cell type specific functions. Physiol. Rev. 85:1159-1204.

37. Unen, J. van, A.D. Stumpf, B. Schmid, N.R. Reinhard, P.L. Hordijk, C. Hoffmann, T.W.J.G. Jr, and J. Goedhart. 2016. A New Generation of FRET Sensors for Robust Measurement of Gai1, Gai2 and Gai3 Activation Kinetics in Single Cells. PLOS ONE. 11:e0146789. 
38. Melnykov, A.V., and K.B. Hall. 2009. Revival of high-order fluorescence correlation analysis: generalized theory and biochemical applications. J. Phys. Chem. B. 113:15629-15638.

39. Dunsing, V., M. Luckner, B. Zühlke, R.A. Petazzi, A. Herrmann, and S. Chiantia. 2018. Optimal fluorescent protein tags for quantifying protein oligomerization in living cells. Sci. Rep. 8:10634.

40. Zacharias, D.A., J.D. Violin, A.C. Newton, and R.Y. Tsien. 2002. Partitioning of lipid-modified monomeric GFPs into membrane microdomains of live cells. Science. 296:913-916.

41. Griesbeck, O., G.S. Baird, R.E. Campbell, D.A. Zacharias, and R.Y. Tsien. 2001. Reducing the Environmental Sensitivity of Yellow Fluorescent Protein: MECHANISM AND APPLICATIONS *. J. Biol. Chem. 276:29188-29194.

42. Landgraf, D., B. Okumus, P. Chien, T.A. Baker, and J. Paulsson. 2012. Segregation of molecules at cell division reveals native protein localization. Nat. Methods. 9:480-482.

43. Rizzo, M.A., G. Springer, K. Segawa, W.R. Zipfel, and D.W. Piston. 2006. Optimization of Pairings and Detection Conditions for Measurement of FRET between Cyan and Yellow Fluorescent Proteins. Microsc. Microanal. 12:238-254.

44. Jeanneteau, F., J. Diaz, P. Sokoloff, and N. Griffon. 2003. Interactions of GIPC with Dopamine D2, D3 but not D4 Receptors Define a Novel Mode of Regulation of G Protein-coupled Receptors. Mol. Biol. Cell. 15:696-705.

45. Kroeze, W.K., M.F. Sassano, X.-P. Huang, K. Lansu, J.D. McCorvy, P.M. Giguere, N. Sciaky, and B.L. Roth. 2015. PRESTO-TANGO: an open-source resource for interrogation of the druggable human GPCR-ome. Nat. Struct. Mol. Biol. 22:362-369.

46. Hendrix, J., T. Dekens, W. Schrimpf, and D.C. Lamb. 2016. Arbitrary-Region Raster Image Correlation Spectroscopy. Biophys. J. 111:1785-1796.

47. Müller, J.D., Y. Chen, and E. Gratton. 2003. Fluorescence correlation spectroscopy. Methods Enzymol. 361:69-92.

48. Levenberg, K. 1944. A method for the solution of certain non-linear problems in least squares. $Q$. Appl. Math. 2:164-168.

49. Marquardt, D.W. 1963. An algorithm for least-squares estimation of nonlinear parameters. J. Soc. Ind. Appl. Math. 11:431-441.

50. Virtanen, P., R. Gommers, T.E. Oliphant, M. Haberland, T. Reddy, D. Cournapeau, E. Burovski, P. Peterson, W. Weckesser, J. Bright, S.J. van der Walt, M. Brett, J. Wilson, K.J. Millman, N. Mayorov, A.R.J. Nelson, E. Jones, R. Kern, E. Larson, C.J. Carey, I. Polat, Y. Feng, E.W. Moore, J. VanderPlas, D. Laxalde, J. Perktold, R. Cimrman, I. Henriksen, E.A. Quintero, C.R. Harris, A.M. Archibald, A.H. Ribeiro, F. Pedregosa, and P. van Mulbregt. 2020. SciPy 1.0: fundamental algorithms for scientific computing in Python. Nat. Methods. 17:261-272. 
bioRxiv preprint doi: https://doi.org/10.1101/2021.11.03.467169; this version posted November 4, 2021. The copyright holder for this preprint (which was not certified by peer review) is the author/funder, who has granted bioRxiv a license to display the preprint in perpetuity. It is made available under aCC-BY 4.0 International license. 


\section{A: G monomer}

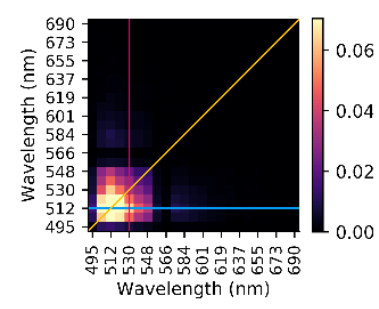

C: R monomer

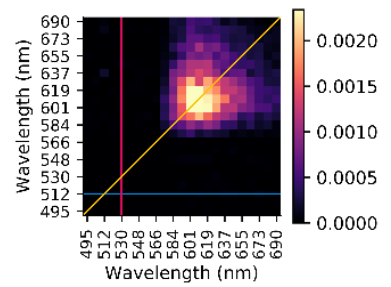

E: GYR trimer

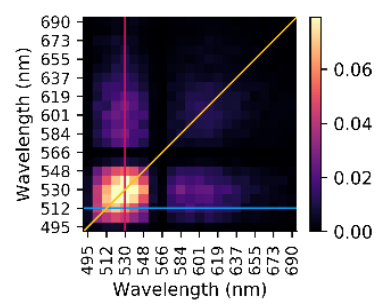

G: G monomer + YR dimer

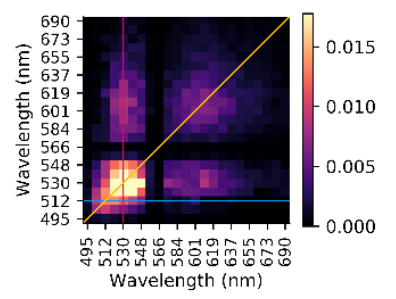

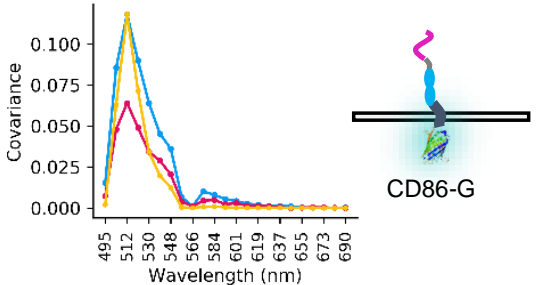
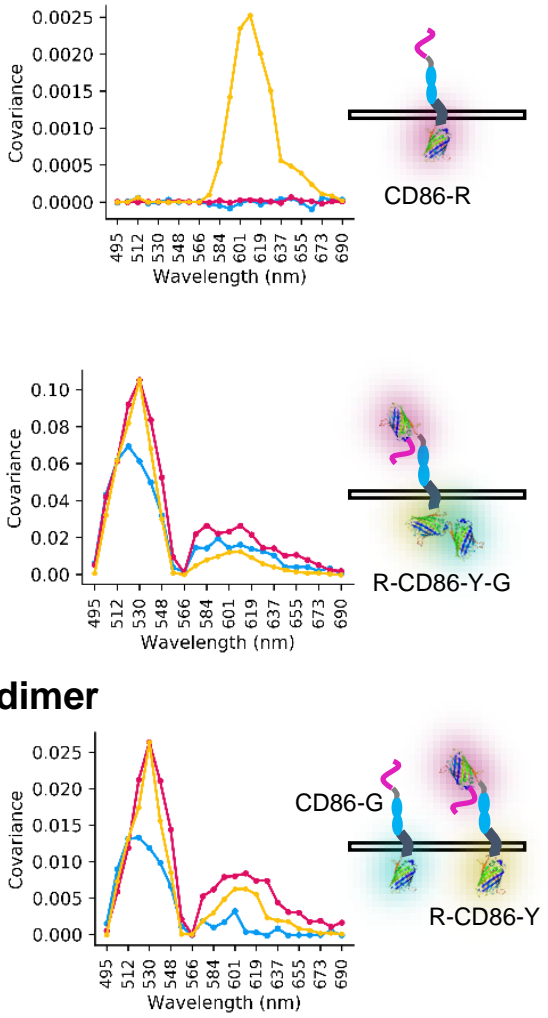

\section{B: Y monomer}
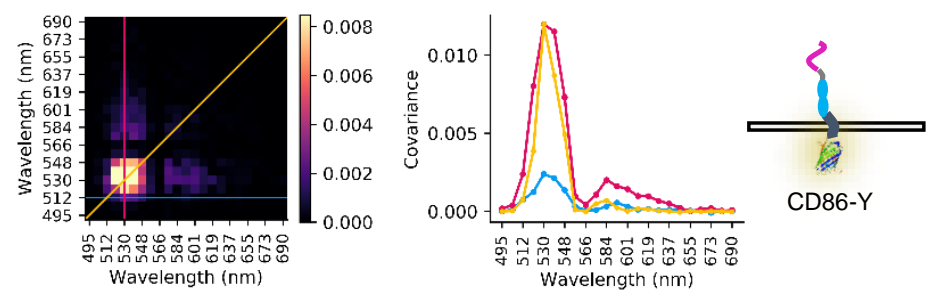

D: G monomer + Y monomer + $\mathbf{R}$ monomer

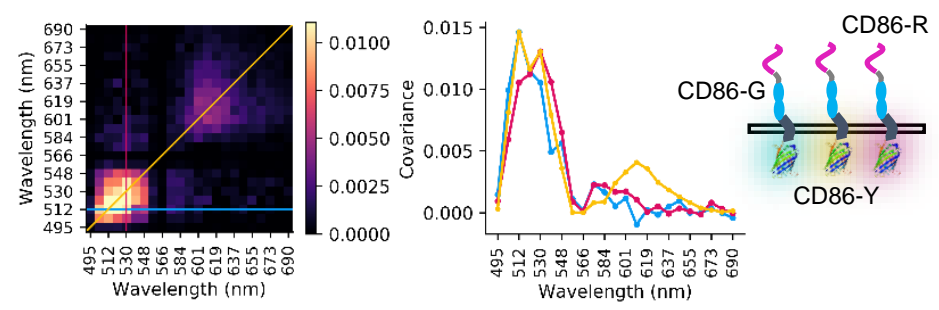

\section{F: GY dimer + R monomer}


\section{H: GR dimer + Y monomer}
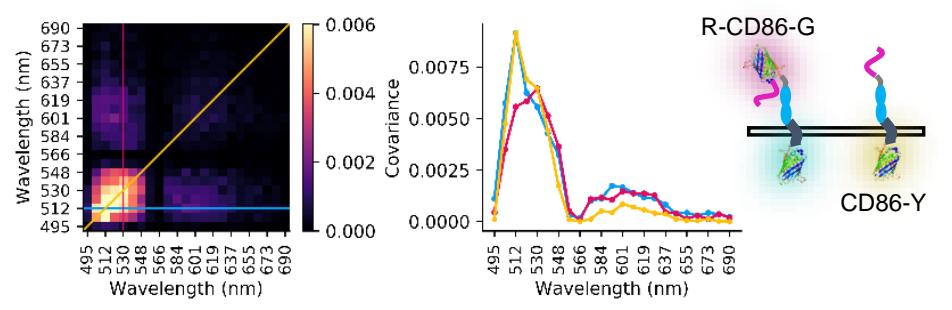

FIGURE 1. Representative covariance matrices $\left(\hat{\kappa}_{[1,1]}\right)$ for cells expressing fluorescent monomers and heteromers in several permutations. A) CD86-G expressed alone. B) CD86-Y. C) CD86-R. D) Triple transfection of CD86-G, CD86-Y, and CD86-R. E) Fluorescent trimer, R-CD86-Y-G. F) Cotransfection of fluorescent heterodimer CD86-Y-G and fluorescent monomer CD86-R. G) Cotransfection of CD86-G and R-CD86-Y. H) Cotransfection of R-CD86-G and CD86-Y. Covariance matrices are on the left of each panel. Middle plots display profiles indicated by lines on covariance matrices. The yellow lines denote the main diagonal. Blue lines denote the line where ordinate is fixed at $512 \mathrm{~nm}$ (peak mEGFP detection). The magenta lines denote where the abscissa is fixed at $530 \mathrm{~nm}$ (peak mEYFP(Q69K) detection). On the right are cartoon representations of the molecular constructs expressed in each sample. 
A. CD86-G CD86-Y CD86-R

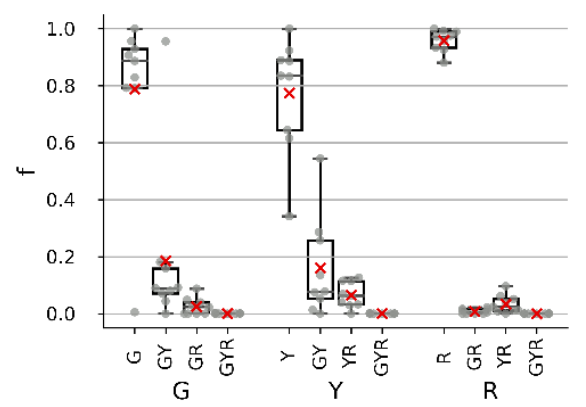

\section{CD86-Y-G CD86-R}



\section{E. R-CD86-G CD86-Y}

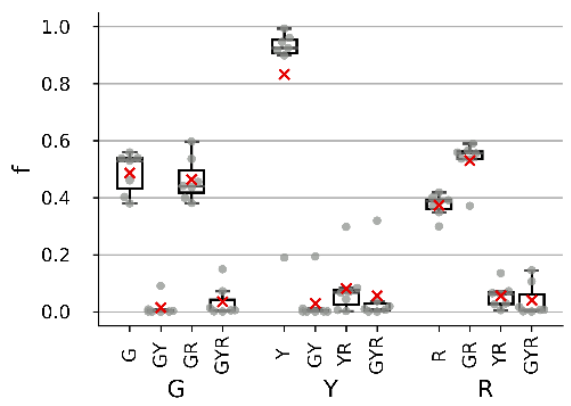

\section{B. R-CD86-Y-G}



D. CD86-G R-CD86-Y

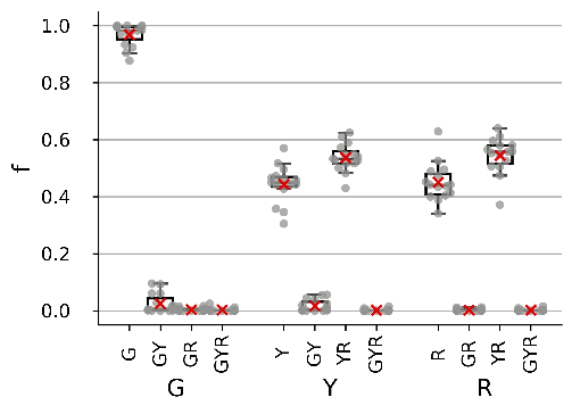

F. Spectrally Resolved RICS

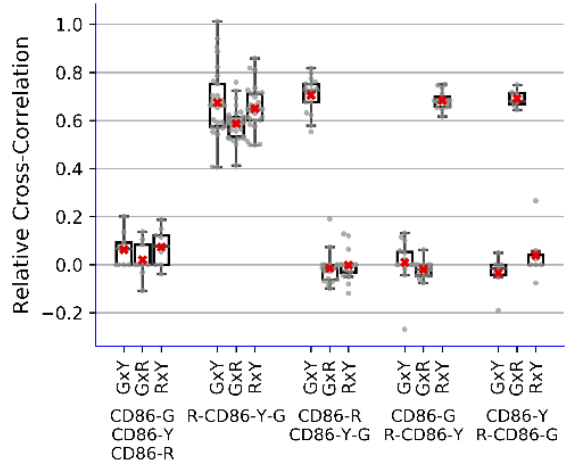

FIGURE 2. Fractional distribution of chromophores among oligomer states determined by fluorescent covariance matrix analysis. Data is from images of HEK 293 cells expressing fluorescent monomers and heteromers. (A) Cotransfection of CD86-G, CD86-Y, and CD86-R. (B) R-CD86-Y-G expressed alone. (C) Cotransfection of CD86-Y-G and CD86-R. (D) Cotransfection of CD86-G and RCD86-Y. (E) Cotransfection of R-CD86-G and CD86-Y. (F) Relative cross-correlations determined by spectrally resolved RICS for all permutations. 
A. GPCR: G-DRD2

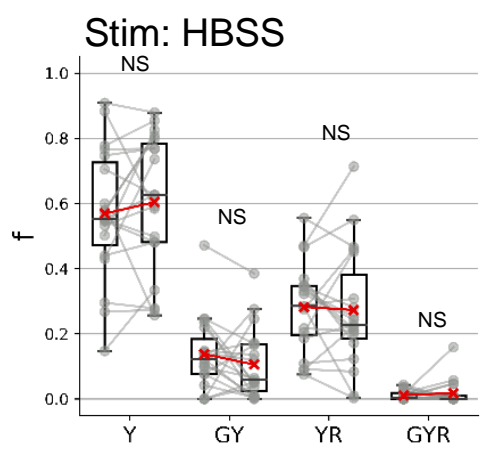

C. GPCR: G-ADRA2A

\section{Stim: HBSS}

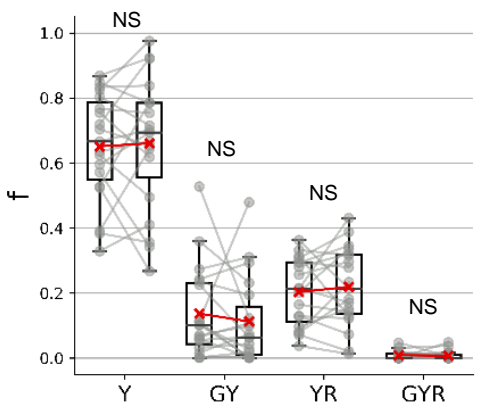

\section{B. GPCR: G-DRD2}

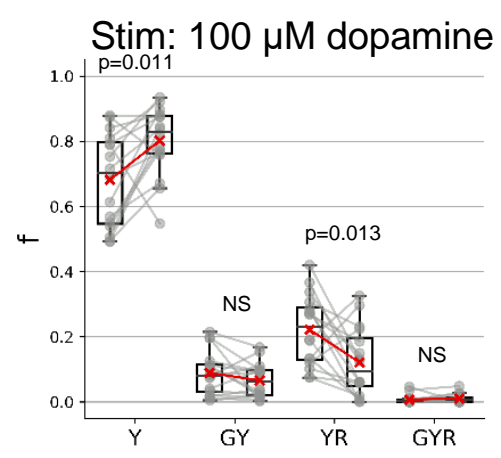

D. GPCR: G-ADRA2A

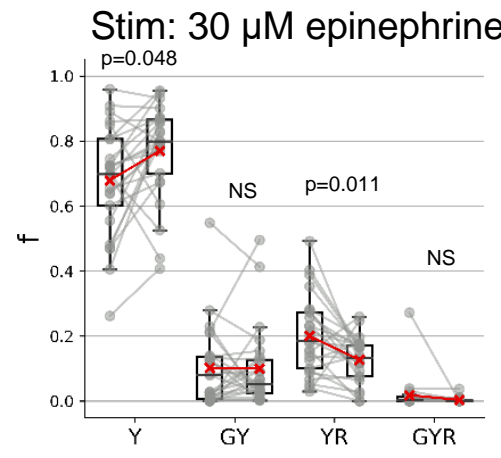

FIGURE 3. Fractional distribution of GNAI1-Y among oligomer states when coexpressed with R-GNB1, R-GNG2, and G-DRD2 or G-ADRA2A determined by covariance matrix analysis. For each state, the left column contains the pre-stimulus fraction, and the right column contains the post-stimulus fraction. A) G-DRD2 is the coexpressed GPCR with the G protein trimer components. Cells were stimulated with additional imaging buffer, HBSS (negative control). B) GPCR G-DRD2 stimulated with $100 \mu \mathrm{M}$ dopamine. C) GPCR G-ADRA2A stimulated with HBSS (negative control). D) G-ADRA2A stimulated with $30 \mu \mathrm{M}$ epinephrine. P-values are the results of two-sided paired t-tests. 
A. GPCR: G-DRD2

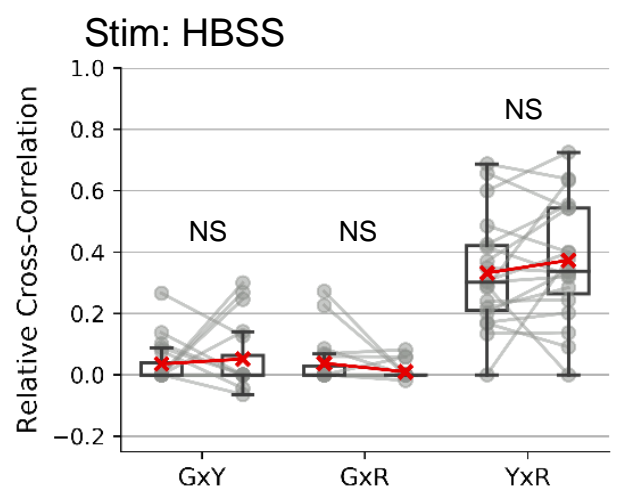

C. GPCR: G-ADRA2A

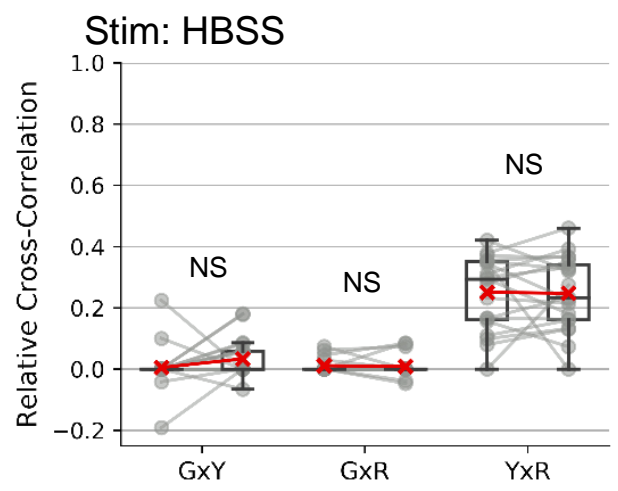

B. GPCR: G-DRD2

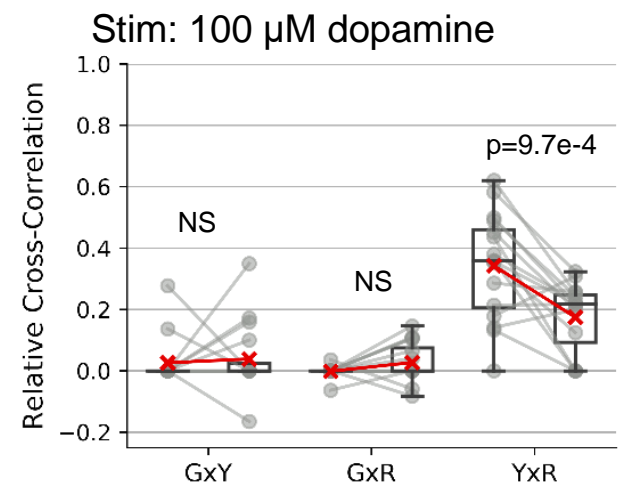

D. GPCR: G-ADRA2A

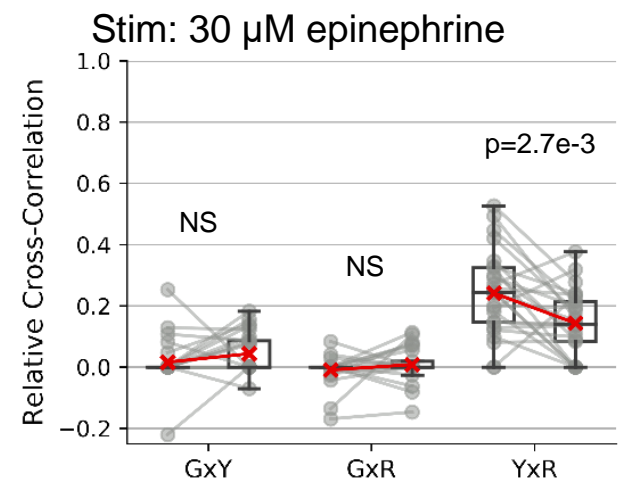

FIGURE 4. Relative cross-correlations determined by spectrally resolved RICS for three components of the $\mathrm{G}$ protein signaling cascade. For each chromophore pairing (i.e. GxY), the left column contains the pre-stimulus relative cross-correlations and the right column contains the post-stimulus crosscorrelations. A) Relative cross-correlations for labeled GPCR G-DRD2 coexpressed with GNAI1-Y and R-GNB1/R-GNG2 stimulated with HBSS (negative control). B) Relative cross-correlations for G-DRD2, Y-GNAI1, and R-GNB1/R-GNG2 stimulated with $100 \mu \mathrm{M}$ dopamine. C) Relative cross-correlations for G-ADRA2A, Y-GNAI1, and R-GNB1/R-GNG2 stimulated with HBSS (negative control). D) Relative cross-correlations for G-ADRA2A, Y-GNAI1, and R-GNB1/R-GNG2 stimulated with $30 \mu \mathrm{M}$ epinephrine. $P$-values were determined by two-sided paired t-tests. NS denotes $p>0.05$. 
A. GPCR: G-DRD2

Stim: HBSS

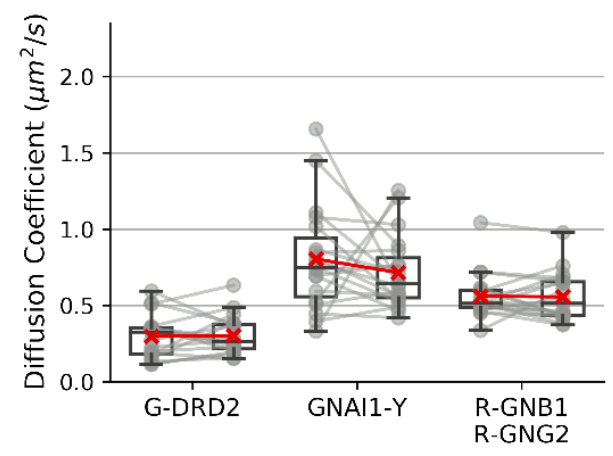

C. GPCR: G-ADRA2A

Stim: HBSS

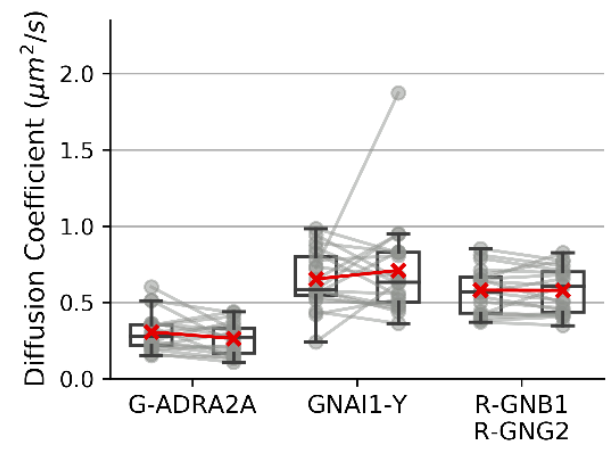

B. GPCR: G-DRD2

Stim: $100 \mu \mathrm{M}$ dopamine

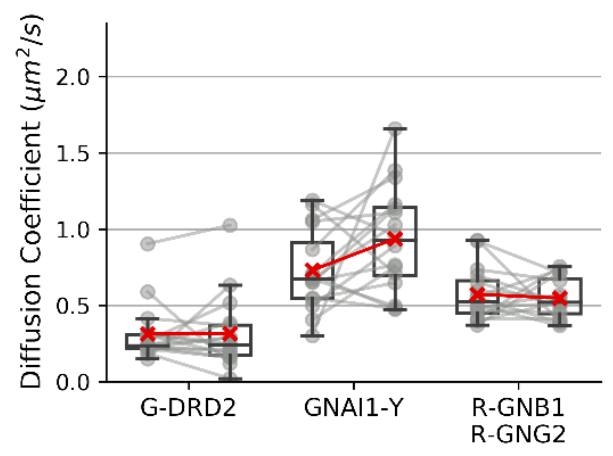

D. GPCR: G-ADRA2A

Stim: $30 \mu \mathrm{M}$ epinephrine

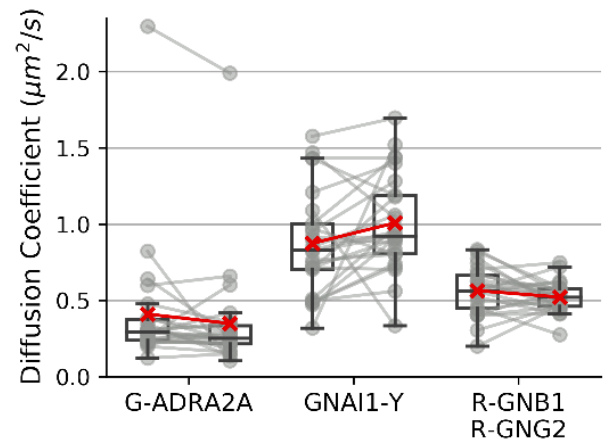

FIGURE 5. Diffusion coefficients for three components of GPCR signaling cascade determined from autocorrelation functions of spectrally resolved RICS. For each component, the left column contains the pre-stimulus diffusion coefficient, and the right column contains the post-stimulus diffusion coefficient. A) G-DRD2, GNAI1-Y, and R-GNB1/R-GNG2 stimulated with HBSS (negative control). B) G-DRD2, GNAI1-Y, and R-GNB1/R-GNG2 stimulated with $100 \mu \mathrm{M}$ dopamine. C) G-ADRA2A, GNAI1-Y, and R-GNB1/R-GNG2 stimulated with HBSS (negative control). D) G-ADRA2A, GNAI1-Y, and RGNB1/R-GNG2 simulated with $30 \mu \mathrm{M}$ epinephrine. No changes between pre- and post-stimulation diffusion coefficients were found to be statistically significant $(p>0.05$, determined by two-sided paired t-test). 
A
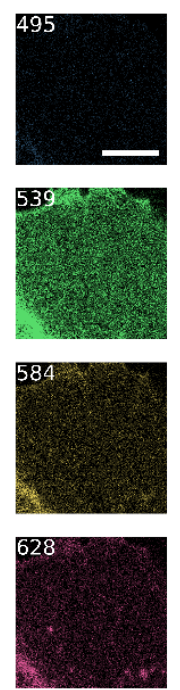


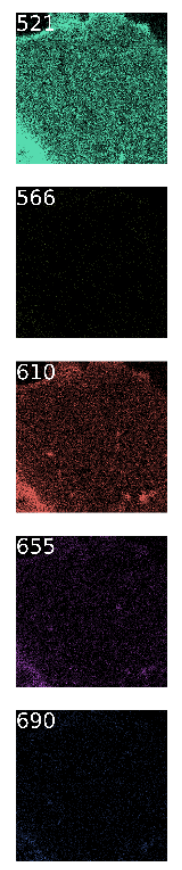

B
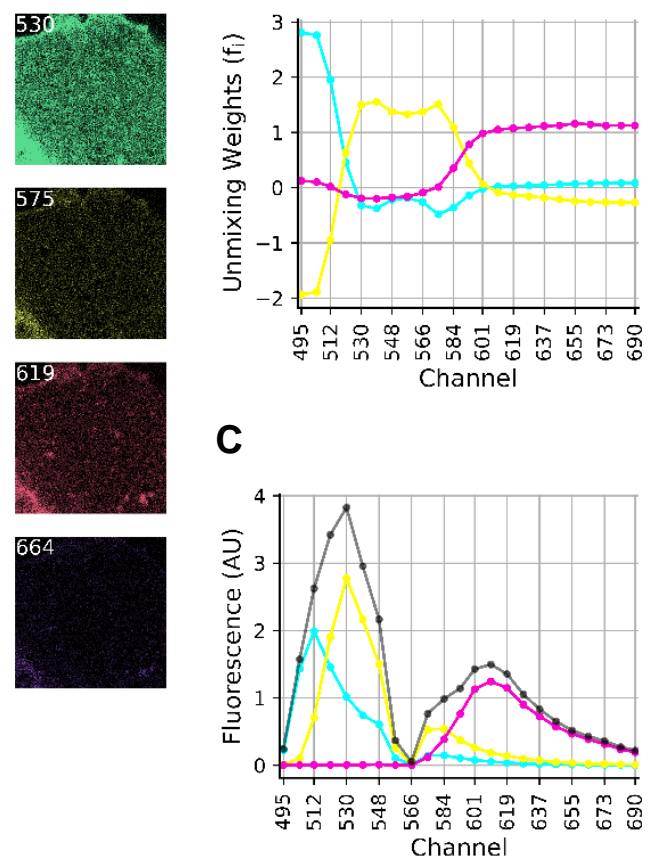

C Channel
D
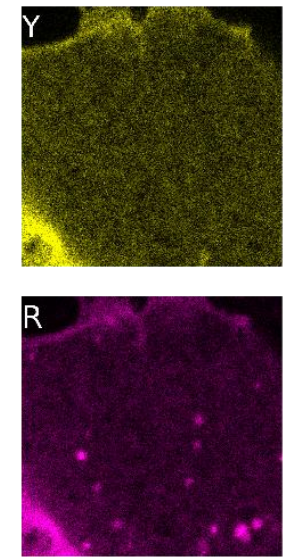

FIGURE S1. Spectral detection and unmixing for covariance matrix analysis and spectrally resolved RICS analysis. A) 23 channel image of the basal plasma membrane of a HEK 293 cell expressing RCD86-Y-G. Labels denote the midpoint wavelength for each channel. B) Weights for spectral unmixing. Cyan, yellow, and magenta lines indicate weights for mEGFP, mEYFP(Q69K), and mCherry2, respectively. C) Detection spectra for image in panel A. The grey line is the composite detection spectrum. Cyan, yellow, and magenta lines represent contributions from mEGFP, $\mathrm{mEYFP}(\mathrm{Q} 69 \mathrm{~K})$ and $\mathrm{mCherry2}$, respectively. D) Spectrally unmixed image corresponding to mEGFP, $\operatorname{mEYFP}(\mathrm{Q} 69 \mathrm{~K})$, and mCherry2. All scale bars are $5 \mu \mathrm{m}$. 

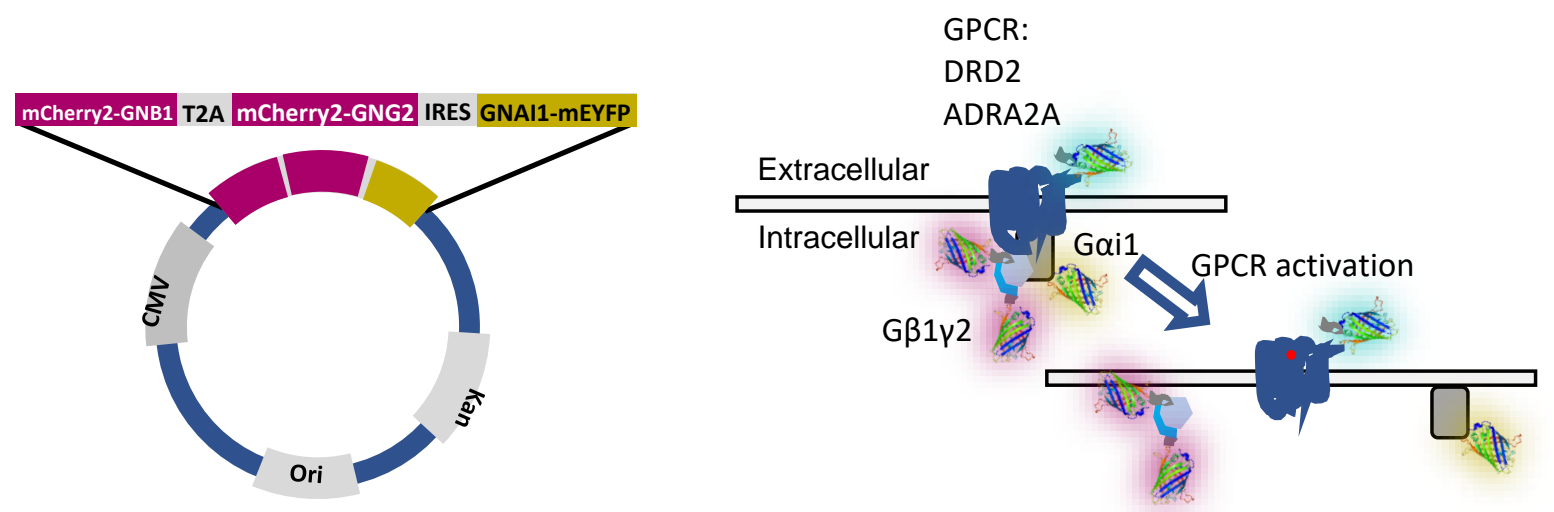

FIGURE S2. Left: Schematic of DNA plasmid used to express three components of the G protein heterotrimer. GNB1 and GNG2 are encoded with mCherry2 tags on their N-termini and separated by a T2A self-cleaving peptide. GNAI1 expression occurs under the control of an internal ribosome entry site and carries an mEYFP(Q69K) tag in the $\alpha b-\alpha c$ loop of GNAI1. Right: Schematic of canonical G protein activation with pre-coupling. A ligand activated GPCR, labeled with mEGFP in this work,

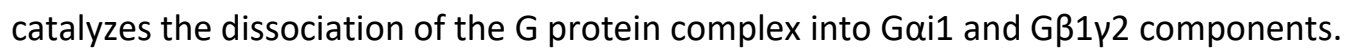




\section{A. GPCR: G-DRD2}

\section{Stim: HBSS}



C. GPCR: G-DRD2

\section{Stim: HBSS}



\section{B. GPCR: G-DRD2}

Stim: $100 \mu \mathrm{M}$ dopamine

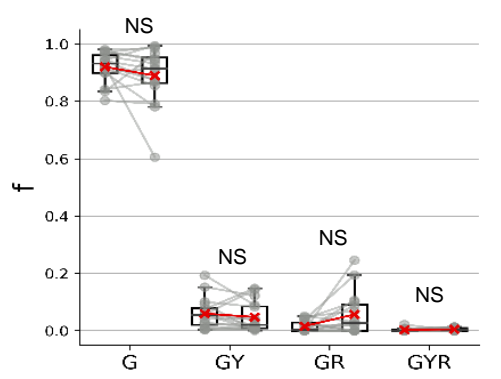

\section{GPCR: G-DRD2}



FIGURE S3. Fractional distributions of G-DRD2 and R-GNB1/R-GNG2 among oligomer states determined by covariance matrix analysis (corresponds with data in Figure $3 \mathrm{~A}-\mathrm{B}$ ). For each state, the left column contains the pre-stimulus fraction, and the right column contains the post-stimulus fraction. A) Fractional oligomer distribution of G-DRD2 stimulated with HBSS (negative control). B) Distribution of G-DRD2 stimulated with $100 \mu \mathrm{M}$ dopamine. C) Distribution of R-GNB1/R-GNG2 stimulated with HBSS (negative control). D) Distribution of R-GNB1/R-GNG2 stimulated with $100 \mu \mathrm{M}$ dopamine. P-values are the results of two-sided paired t-tests. NS denotes $p>0.05$. 
A. GPCR: G-ADRA2A

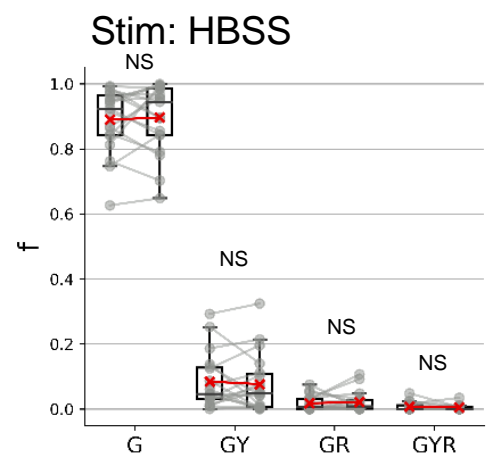

C. GPCR: G-ADRA2A

Stim: HBSS

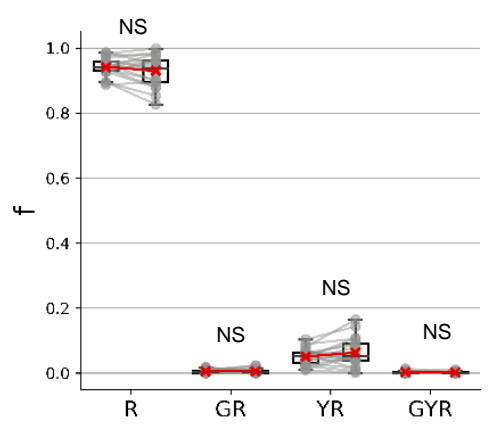

B. GPCR: G-ADRA2A

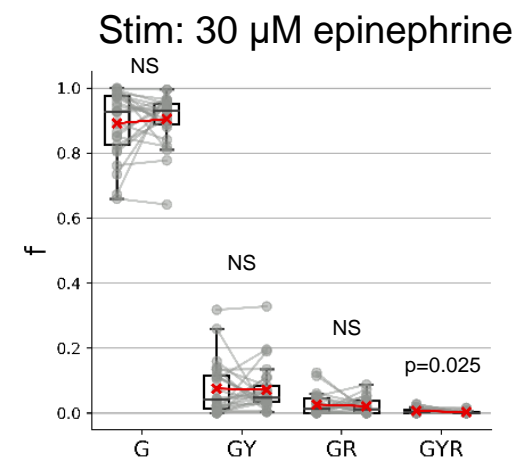

D. GPCR: G-ADRA2A

Stim: $30 \mu \mathrm{M}$ epinephrine

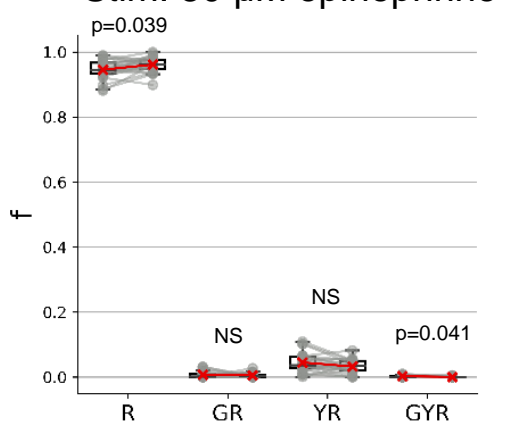

FIGURE S4. Fractional distributions of G-ADRA2A and R-GNB1/R-GNG2 among oligomer states determined by covariance matrix analysis (corresponds with data in Figure $3 \mathrm{C}-\mathrm{D}$ ). For each state, the left column contains the pre-stimulus fraction, and the right column contains the post-stimulus fraction. A) Fractional oligomer distribution of G-ADRA2A stimulated with HBSS (negative control). B) Distribution of G-ADRA2A stimulated with $30 \mu \mathrm{M}$ epinephrine. C) Distribution of R-GNB1/R-GNG2 stimulated with HBSS (negative control). D) Distribution of R-GNB1/R-GNG2 stimulated with $30 \mu \mathrm{M}$ epinephrine. P-values are the results of two-sided paired t-tests. NS denotes $p>0.05$. 
bioRxiv preprint doi: https://doi.org/10.1101/2021.11.03.467169; this version posted November 4, 2021. The copyright holder for this preprint (which was not certified by peer review) is the author/funder, who has granted bioRxiv a license to display the preprint in perpetuity. It is made available under aCC-BY 4.0 International license.

Table S1. Primers for subcloning experiments.

\begin{tabular}{|c|c|c|}
\hline Forward Primer $\left(5^{\prime}->3^{\prime}\right)$ & Reverse Primer (5'->3') & Purpose \\
\hline TAAAGCGGCCGCGACTCTAG & AGTAGCGACCGGGGTTGC & $\begin{array}{l}\text { Linearize CD86-EGFP about EGFP coding region for FP swaps } \\
\text { against EGFP }\end{array}$ \\
\hline $\begin{array}{l}\text { ACCCCGGTCGCTACTATGGTGA } \\
\text { GCAAGGGCGAGG }\end{array}$ & $\begin{array}{l}\text { GTCGCGGCCGCTTTACTTGTA } \\
\text { CAGCTCGTCCATGCC }\end{array}$ & $\begin{array}{l}\text { Amplification of mEGFP, mEYFP(Q69K) or mCherry2 for insertion } \\
\text { against EGFP in CD86-EGFP to create CD86-G, CD86-Y, CD86-R }\end{array}$ \\
\hline TCCGGAATGGCCCCTCTG & GGTGGCGACCGGAATCTTC & $\begin{array}{l}\text { Linearize EGFP-CD86-mApple about EGFP coding region for } \\
\text { swaps against mApple }\end{array}$ \\
\hline $\begin{array}{l}\text { TGAAGATTCCGGTCGCCACCAT } \\
\text { GGTGAGCAAGGGCGAGG }\end{array}$ & $\begin{array}{l}\text { TTCAGAGGGGCCATTCCGGA } \\
\text { CTTGTACAGCTCGTCCATGCC }\end{array}$ & $\begin{array}{l}\text { Amplification of mCherry2 for insertion against EGFP in EGFP- } \\
\text { CD86-mApple. One step in creating R-CD86-G and R-CD86-Y. }\end{array}$ \\
\hline TTCGCTGCGCTGCTGGCAAC & TCCCTCCCACTGGGGCAC & $\begin{array}{l}\text { Linearize CD86-G or R-CD86-G about position in C-terminal linker } \\
\text { region for insertion of an additional FP coding region }\end{array}$ \\
\hline $\begin{array}{l}\text { CCCCAGTGGGAGGGAATGGTG } \\
\text { AGCAAGGGCGAGG }\end{array}$ & $\begin{array}{l}\text { CAGCAGCGCAGCGAACTTGT } \\
\text { ACAGCTCGTCCATGCC }\end{array}$ & $\begin{array}{l}\text { Amplification of mEYFP(Q69K) for insertion into C-terminal linker } \\
\text { region of CD86-G or R-CD86-G to create CD86-Y-G or R-CD86-Y-G }\end{array}$ \\
\hline $\begin{array}{l}\text { CGTCAGATCCGCTAGCGCTACC } \\
\text { GGTCGCCACCA }\end{array}$ & $\begin{array}{l}\text { TGGGAGTAAGTCATGAGCTC } \\
\text { CTGGTCAGCACGAA }\end{array}$ & $\begin{array}{l}\text { Amplification of R-GNB1 for insertion into GNB1-T2A-R-GNG2- } \\
\text { IRES-GNAI1-Y linearized by digestion with Nhel and Sacl to } \\
\text { produce R-GNB1-T2A-R-GNG2-IRES-GNAI1-Y }\end{array}$ \\
\hline TCCGGACTCAGATCTATGGCCA & ACCGGTAGGGCCGGGATT & $\begin{array}{l}\text { Linearize R-GNB1-T2A-cpVenus-GNG2-IRES-GNAI1-mTurquoise2 } \\
\text { about cpVenus }\end{array}$ \\
\hline $\begin{array}{l}\text { AATCCCGGCCCTACCGGTATG } \\
\text { GTGAGCAAGGGCGAGG }\end{array}$ & $\begin{array}{l}\text { CATAGATCTGAGTCCGGACTT } \\
\text { GTACAGCTCGTCCATGCC }\end{array}$ & $\begin{array}{l}\text { Amplify for insertion against cpVenus in R-GNB1-T2A-cpVenus- } \\
\text { GNG2-IRES-GNAI1-mTurquoise2 to create R-GNB1-T2A-R-GNG2- } \\
\text { IES-GNAI1-mTurquiose2 }\end{array}$ \\
\hline $\begin{array}{l}\text { TTATGACCGCGACCGGTTCTAT } \\
\text { GGTGAGCAAGGGCGAGG }\end{array}$ & $\begin{array}{l}\text { ATGACGCCGGCGAGTTCACC } \\
\text { AGTGATCCCGGCGGCGGTCA } \\
\text { CG }\end{array}$ & $\begin{array}{l}\text { Amplification of mEYFP(Q69K) for insertion into GNAI1- } \\
\text { mTurquiose2 linearized by Agel digestion against mTurquiose2 }\end{array}$ \\
\hline $\begin{array}{l}\text { GAGCACCCAGTCCAAGCTGAG } \\
\text { CAAAGAC }\end{array}$ & $\begin{array}{l}\text { AGGTAGTGGTTGTCGGGCAG } \\
\text { CAGC }\end{array}$ & $\begin{array}{l}\text { Introduce A206K mutation into EGFP in EGFP-DRD2 to create G- } \\
\text { DRD2 }\end{array}$ \\
\hline TGACTCTGCTGCCTGCCC & GTACAGCTCGTCCATGCCG & Linearize G-DRD2 about DRD2 \\
\hline $\begin{array}{l}\text { GGCATGGACGAGCTGTACATG } \\
\text { TTCCGGCAGGAGCAGC }\end{array}$ & $\begin{array}{l}\text { GGGCAGGCAGCAGAGTCAAA } \\
\text { CGATTCGTTTCCTATCGCCTC }\end{array}$ & $\begin{array}{l}\text { Amplify ADRA2A for insertion against DRD2 in G-DRD2 to create } \\
\text { G-ADRA2A }\end{array}$ \\
\hline
\end{tabular}

